The long non-coding RNA landscape in juvenile myelomonocytic leukemia

Juvenile myelomonocytic leukemia (JMML) is a rare and aggressive myelodysplastic and myeloproliferative disorder of early childhood. It is characterized by proliferation of granulocytic and monocytic cells. ${ }^{1}$ Currently, hematopoietic stem cell transplantation (HSCT) is the standard of care and results in long-term overall survival (OS) of only $50-60 \%{ }^{2}$ In approximately $90-95 \%$ of patients, hyperactivation of the RAS/MAPK pathway can be observed through typical mutations in NRAS and KRAS (20-25\%), PTPN11 (35\%), NF1 (10-15\%) or CBL (10-15\%) genes. This underlying molecular defect can in part be used to guide patient management (e.g. no HSCT in patients with mutations in $C B L)^{2}$ Additional somatic mutations, including mutations in SETBP1, JAK3 and members of the polycomb repressive complex 2 (PRC2), are associated with worse outcome. ${ }^{3,4}$ In addition, LIN28B overexpression and DNA hypermethylation profiles can identify JMML patients with a more aggressive clinical course. $^{5-7}$

The non-coding transcriptome consists of a variety of different RNA types such as tRNAs, snoRNAs, microRNAs, circRNAs and long non-coding RNAs (lncRNAs). LncRNAs are a class of RNA genes with a minimum length of 200 nucleotides. $^{8}$ Although only a fraction of known lncRNAs have been functionally characterized, there is growing evidence of their involvement in a variety of biological processes, human diseases and malignancies.

To explore lncRNA expression in JMML, RNA from isolated mononuclear cell preparations from 44 previously untreated JMML patients (median age: 2.02 years) and 7 bone marrow samples from healthy controls (siblings screened for transplantation; median age 6.83 years) was profiled on a custom designed Agilent (8x60K) microarray platform described by Volders et al. ${ }^{8}$ The dataset (GEO Series accession n. GSE71449) had been previously used in an mRNA expression analysis, leading to the identification of overexpression of LIN $28 B$ in a fetal-like subgroup of JMML patients. ${ }^{5}$ Details of patients' characteristics can be found in the Online Supplementary Table S1

Only probes $(n=23,728)$ representing known lncRNAs were withheld for further analyses. Therefore, the probes were mapped against the most up-dated version of different IncRNA databases, including Incipedia (www.Incipedia.org), Hugo Gene Nomenclature Committee (HGNC), and Long Noncoding RNA Database v.2.0 (www.lncrnadb.org). The Incipedia 3.1 annotations for IncRNAs have been used throughout this manuscript. Differential gene expression analysis, with samr package (R BioConductor) and BRB-Array tools, revealed a total of 300 differentially expressed lncRNAs (adjusted $P<0.05$; FDR $<0.10$ ) between JMML patients and controls, of which 15 up-regulated and 285 down-regulated in JMML patients (Figure $1 \mathrm{~A}$ and $\mathrm{B}$ and Online Supplementary Table S2). The 5 most up-regulated

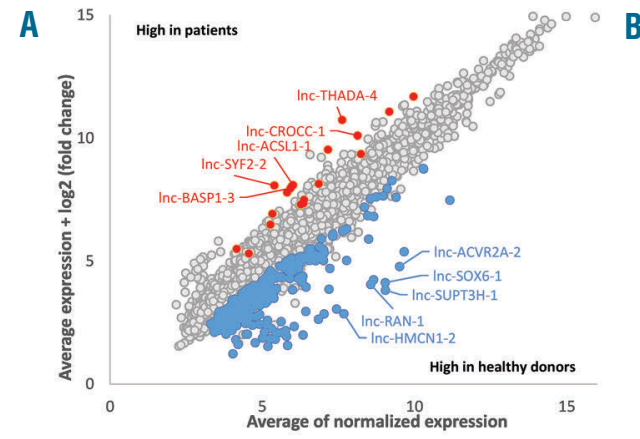

B
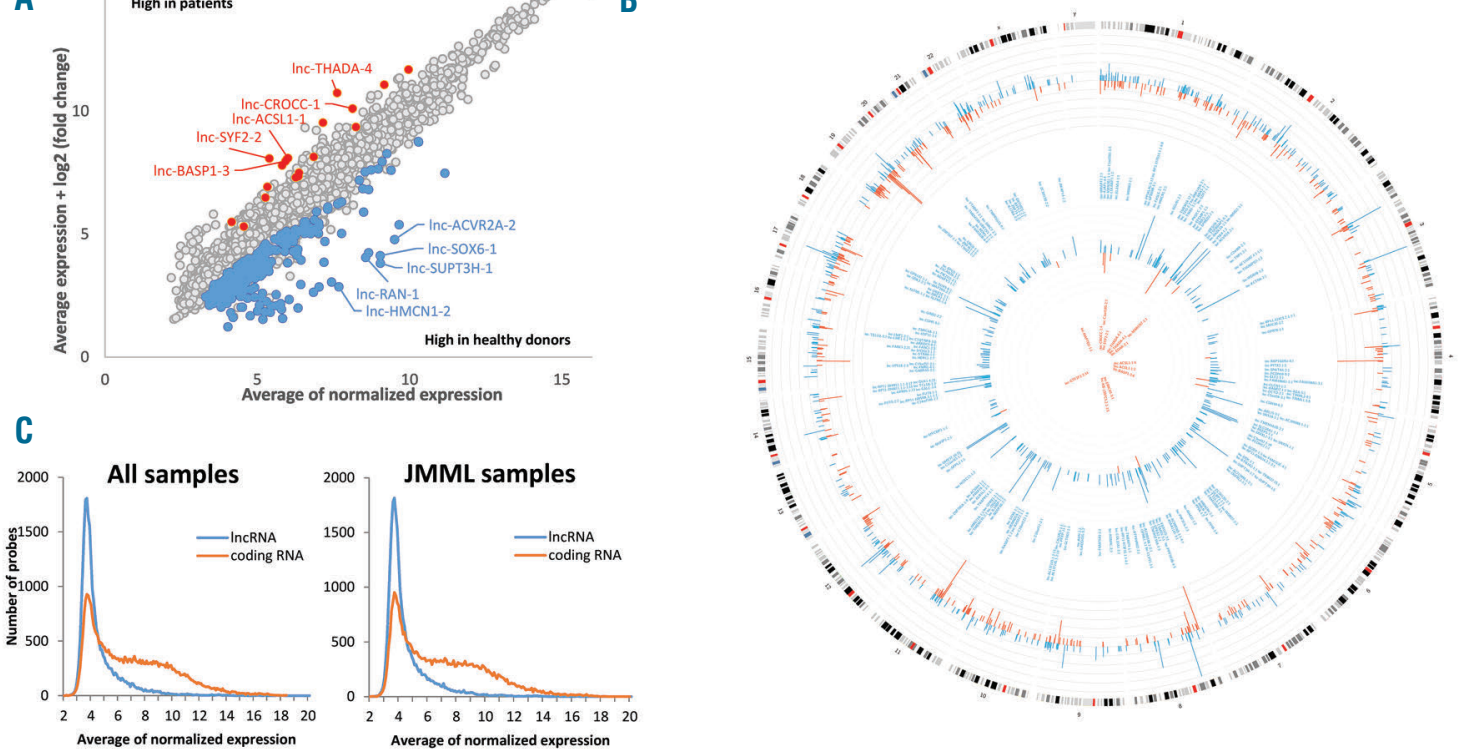

Figure 1. Long non-coding RNA transcriptome in juvenile myelomonocytic leukemia (JMML). (A) Diagonal plot representing the average expression versus the log2 fold change added to the average expression of IncRNAs when comparing JMML $(n=44)$ to healthy donors ( $n=7$ ). IncRNAs significantly up-regulated in patients are represented in red and IncRNAs significantly down-regulated in blue. The names of the top 5 up- and down-regulated IncRNAs based on log2 fold change are indicated. (B) Circos plot showing IncRNAs and mRNAs on human chromosomes. From the outside in, the first layer of the Circos plot represents a chromosome map of the human genome. Black and white bars are chromosome cytobands, and red bars represent centromeres. The second circle shows all differentially expressed coding genes when comparing JMML patients with healthy donors, and the inner circle shows all differentially expressed IncRNAs. Upregulated InCRNA and mRNA genes are represented in red and down-regulated genes in blue. The height of the bars is representative for the fold change between JMML and healthy donors. Names of IncRNA genes with fold change of more than 2 between JMML and healthy donors are indicated between the circles with mRNA and IncRNA genes for the down-regulated IncRNAs and in the middle of the plot for the up-regulated IncRNAs. (C) Density plots representing the average of the normalized expression of all IncRNAs and mRNAs detected by the array in all samples (left) and in JMML samples only (right). 
IncRNAs based on log2 fold change were $\operatorname{lnc}-T H A D A-4$, Inc-SYF2-2, Inc-BASP1-3, Inc-ACSL1-1 and Inc-CROCC-1, whereas $\operatorname{lnc}-S U P T 3 H-1, \operatorname{lnc}-S O X 6-1, \operatorname{lnc}-H M C N 1-2$, lnc$A C V R 2 A-2$ and $\operatorname{lnc}-R A N-1$ were the top 5 most downregulated IncRNAs. As shown in other disease entities, the mean expression level of IncRNAs was nearly 2-fold lower as compared to mRNA $(P<0.0001)$ (Figure $1 \mathrm{C})$. To exclude the impact of differences in cellular composition between JMML and healthy controls, we compared our dataset with an IncRNA expression atlas of flow cytometry sorted blood cell populations (Online Supplementary Table S3). ${ }^{10}$ This analysis revealed 2 of 15 differentially up-regulated IncRNAs in JMML to be specific for granulocytic lineage (lnc-ACSL1-1 and $\ln c-B A S P 1-3)$ and none for monocytic differentiation. Conversely, one monocytic specific lncRNA showed significant downregulation in JMML patients (Inc-AL713998.1-2).

To explore the functional characteristics of differentially expressed IncRNAs, we used a guilt-by-association approach, based on the hypothesis that non-coding and protein coding genes belonging to the same biological pathways are co-ordinately regulated. Spearman's rho values were calculated between IncRNAs of interest and all protein coding genes on the array. The resulting ranked gene lists served as inputs for pre-ranked gene-set enrichment analysis (GSEA), ${ }^{11}$ using the MSigDB.v.6.0 C2 collection and FDR $<0.1$. This revealed an overlap between the 15 curated gene sets most positively correlated with the top 5 up-regulated IncRNAs (Figure 2 and Online Supplementary Table S4). Using the same approach for the top 5 down-regulated lncRNAs, a strong overlap both in gene sets positively correlated with these IncRNAs, as well as negatively correlated was observed (Figure 2 and Online Supplementary Table S4). Interestingly, several gene sets showed positive correlation with up-regulated IncRNAs as well as negative correlation with down-regulated IncRNAs, suggesting a common pathway. Notably, these gene sets are related to myeloid cell development, dendritic cell maturation, neutrophil function at skin wound, systemic lupus erythematosus, and different acute myeloid leukemia (AML) phenotypes.

Extensive cytogenetic and mutational data were available for our patient cohort and we evaluated whether the expression of IncRNAs was associated with specific JMML subgroups based on clinical, cytogenetic and mutational characteristics. First, patients with elevated fetal hemoglobin $(\mathrm{HbF})$ levels for age were compared with patients with normal HbF levels. Only 8 IncRNAs were found differentially expressed between these two

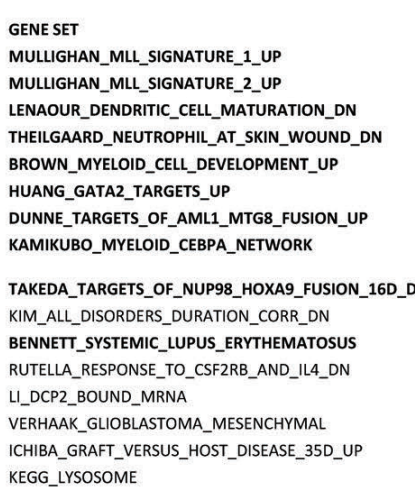

Gene sets correlated with top 5 upregulated IncRNAs in JMML

\begin{tabular}{|c|c|c|c|c|}
\hline InC-THADA-4 & InC-SYF2-2 & InC-BASP1-3 & InC-ACSL1-1 & InC-CROCC-1 \\
\hline+ & + & + & & + \\
\hline \multirow[t]{2}{*}{+} & + & + & & + \\
\hline & + & + & + & \\
\hline \multirow[t]{7}{*}{+} & + & + & & + \\
\hline & + & + & + & + \\
\hline & + & + & & \\
\hline & + & + & & + \\
\hline & + & + & & \\
\hline & + & + & & + \\
\hline & + & + & + & \\
\hline & & & & \\
\hline & & & & \\
\hline & & & & \\
\hline
\end{tabular}

\section{GENE SET}

REACTOME_OLFACTORY_SIGNALING_PATHWAY KEGG_OLFACTORY_TRANSDUCTION MIKKELSEN_MCV6_HCP_WITH_H3K27ME3 MIKKELSEN_NPC_HCP_WITH_H3K27ME3 VECCHI_GASTRIC_CANCER_EARLY_DN MEISSNER_BRAIN_HCP_WITH_H3K27ME3 KONDO_PROSTATE_CANCER_WITH_H3K27ME3 THUM_MIR21_TARGETS_HEART_DISEASE_UP NIKOLSKY_BREAST_CANCER_19Q13.1_AMPLICON SU_KIDNEY

MAHADEVAN_IMATINIB_RESISTANCE_UP REACTOME_SIGNALING_BY_BMP STAEGE_EWING_FAMILY_TUMOR KEGG_STEROID_HORMONE_BIOSYNTHESIS YAMASHITA_LIVER_CANCER_WITH_EPCAM_DN WEBER METHYLATED_ICP_IN_FIBROBLAST KEGG_MATURITY_ONSET_DIABETES_OF_THE_YOUNG
Gene sets anticorrelated with top 5 downregulated IncRNAs in JMML InC-SUPT3H-1 InC-SOX6-1 InC-HMCN1-2 InC-ACVR2A-2 InC-RAN-1

\begin{tabular}{|c|c|c|c|c|c|c|c|c|c|}
\hline \multicolumn{5}{|c|}{ Gene sets anticorrelated with top 5 upregulated IncRNAs in JMML } & \multicolumn{5}{|c|}{ Gene sets correlated with top 5 downregulated IncRNAs in JMML } \\
\hline Inc-THADA-4 & Inc-SYF2-2 & InC-BASP1-3 & InC-ACSL1-1 & Inc-CROCC-1 & Inc-SUPT3H-1 & Inc-SOX6-1 & Inc-HMCN1-2 & InC-ACVR2A-2 & Inc-RAN-1 \\
\hline - & - & - & & - & + & + & + & + & + \\
\hline - & - & - & & - & + & + & + & + & + \\
\hline - & - & & & - & & & & & \\
\hline \multirow[t]{3}{*}{-} & - & & & - & + & + & & & + \\
\hline & - & - & & - & & & & & \\
\hline & - & - & & & + & + & + & + & + \\
\hline \multirow[t]{11}{*}{-} & & & & - & + & + & + & + & + \\
\hline & & & - & & + & & + & + & + \\
\hline & & & - & & + & + & + & + & + \\
\hline & & & & & + & + & + & + & + \\
\hline & & & & & + & + & + & + & + \\
\hline & & & & & & + & + & + & + \\
\hline & & & & & & + & + & + & + \\
\hline & & & & & + & & + & + & + \\
\hline & & & & & + & + & + & + & \\
\hline & & & & & + & + & & + & + \\
\hline & & & & & + & + & + & & + \\
\hline
\end{tabular}

Figure 2. Schematic overview of the overlap between curated gene sets positively and negatively correlating with the top 5 up- and down-regulated IncRNAs. The overlap between curated gene sets positively and negatively correlating with the top 5 up-regulated IncRNAs (Inc-THADA-4, Inc-SYF2-2, Inc-BASP1-3, IncACSL1-1 and Inc-CROCC-1) and the top 5 down-regulated IncRNAs (Inc-SUPT3H-1, Inc-SOX6-1, Inc-HMCN1-2, Inc-ACVR2A-2 and Inc-RAN-1) in juvenile myelomonocytic leukemia (JMML) compared to healthy controls. Only gene sets with overlap in 3 or more IncRNAs are presented. The green '+' symbol indicates a positive correlation between the gene set and the IncRNA, and the red "-' symbol indicates a negative correlation between the gene set and the IncRNA. Gene sets (anti-)correlating both with up-regulated and down-regulated IncRNAs are written in bold. Marked overlap between these gene sets can be observed suggesting a common pathological pathway. 
groups, all up-regulated in the group with normal $\mathrm{HbF}$ levels for age (adjusted $P<0.05)$ (Online Supplementary Table S5). Given the strong downregulation of IncC2orf54-1 and lnc-ATP6VOD2-2 in JMML patients with elevated $\mathrm{HbF}$ for age (log fold change -7.4 and -2.8, respectively), we predicted that these IncRNA would be anti-correlated with genes involved in $\mathrm{HbF}$ regulation. Indeed, HBG1 (Spearman rho value $(\rho):-0.63$ and -0.61 ), HBBP1 (Hemoglobin Subunit Beta Pseudogene 1; $\rho$ : -0.60 and -0.41), HBE1 ( $\rho$ : -0.37 and -0.45) and LIN28B ( $\rho$ : 0.58 and -0.70$)$, belonged to the top anti-correlated protein coding genes, suggesting that both $\operatorname{lnc}-\mathrm{C} 2$ orf54-1 and Inc-ATP6VOD2-2 could be part of a molecular network controlling the formation of $\mathrm{HbF}$. Moreover, IncATP6VOD2-2 is associated with event-free survival (EFS) in cytogenetically normal AML and is down-regulated in patients with mutations in RUNX1. ${ }^{12}$ Subsequently, patients with monosomy 7 were compared to patients with a normal karyotype. To avoid confounding effects due to RAS gene mutations, a 2-class analysis (monosomy 7 vs. normal karyotype) blocking for $R A S$ mutations through a randomized block design was applied. ${ }^{13}$ As such, different $R A S$ mutations are considered one group (block), eliminating variability not of interest in this specific analysis. ${ }^{13}$ This analysis revealed a total of 36 lncRNAs significantly up-regulated in the monosomy 7 group and 5 lncRNAs down-regulated. Of note, 6 of 8 IncRNAs (lnc-C2orf54-1, PRINS, Inc-ATP6VOD2-2, IncCCT8-1, lnc-SH3BGRL2-1, lnc-FRMD4A-1) down-regu- lated in patients with elevated $\mathrm{HbF}$ levels for age were significantly up-regulated in JMML patients with monosomy 7 . This observation corresponds with the established inverse relation between monosomy 7 and elevated $\mathrm{HbF}$ levels in JMML patients.

Next, we performed pairwise analyses comparing patients with PTPN11 ( $\mathrm{n}=18)$, KRAS $(\mathrm{n}=11), N R A S(\mathrm{n}=8)$, NF1 ( $\mathrm{n}=4)$ mutations or without any of the above mutations (quadruple negative; $n=3$ ) to all other patients (Online Supplementary Table S5). Patients harboring a $K R A S$ mutation showed 26 differentially expressed IncRNAs (17 up-regulated and 9 down-regulated) compared to JMML patients without this mutation. NF1 mutated patients were characterized by 62 down-regulated IncRNAs whereas none were significantly up-regulated. PTPN11 mutated patients were marked by differential expression of 8 IncRNAs (6 up-regulated and 2 downregulated) and $4 \operatorname{lncRNAs}$ were differentially expressed in NRAS mutated JMML (all up-regulated). Remarkably, in quadruple negative patients, only one IncRNA, lnc$A T P 10 A-2$, was differentially up-regulated with high fold change $(\mathrm{FC}=14.4)$. This IncRNA is anti-correlated with $B A P 1$, a key regulator of many cancer-associated pathways.

Finally, we studied differentially expressed lncRNAs between JMML patients with and without LIN28B overexpression. Sixty-one IncRNAs were differentially expressed of which 60 were up-regulated in JMML patients with LIN28B overexpression (Online
A

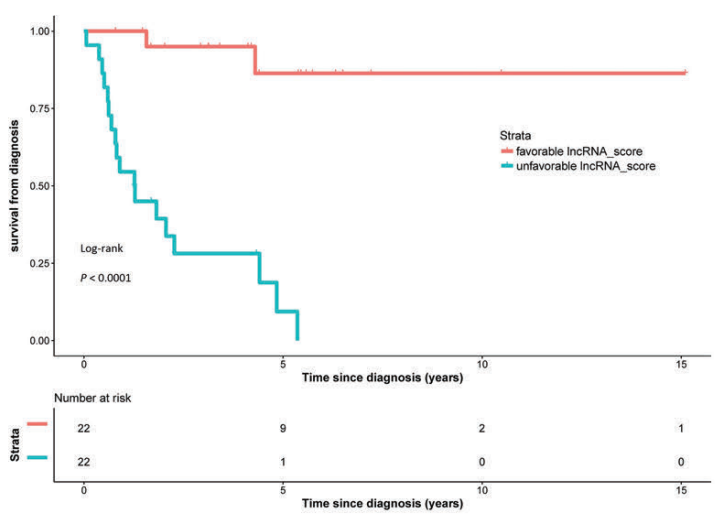

C

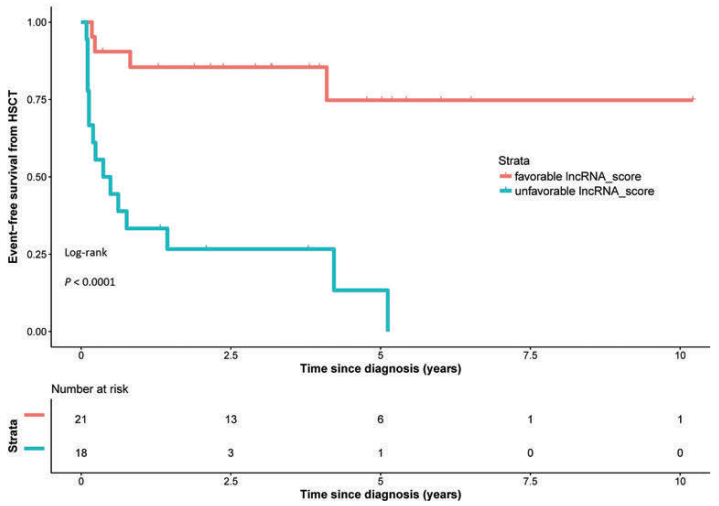

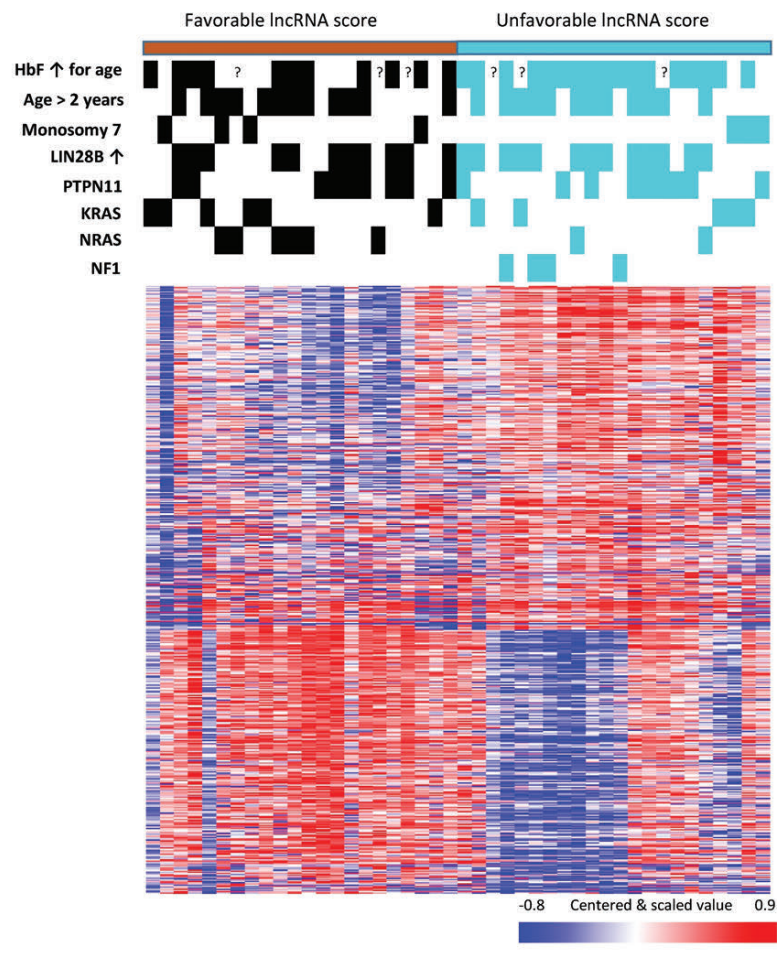

Figure 3. Stratification of juvenile myelomonocytic leukemia (JMML) patients based on IncRNA score. (A and C) Kaplan-Meier curve for overall survival (OS) and event-free survival (EFS) after hematopoietic stem cell transplantation (HSCT) from JMML diagnosis for patients with favorable and unfavorable IncRNA score. (B) Heatmap displaying differential gene expression ( $n=549$ coding genes with an absolute fold change of more than 2; 238 genes up-regulated in patients with favorable IncRNA score and 311 genes up-regulated in patients with unfavorable IncRNA score) between JMML patients with favorable and unfavorable IncRNA score. Clinical annotation for "genotype" (somatic mutations in PTPN11, KRAS, NRAS and NF1; LIN28B overexpression and karyotype) and some clinical characteristics [age group and fetal hemoglobin ( $\mathrm{HbF}$ ) level for age] are depicted on top of the heatmap. 
Supplementary Table S5). Interestingly, both MEG3 and MEG8 were up-regulated in LIN28B overexpression and are known to be involved in regulation of pluripotence and fetal development. ${ }^{14}$

As the prognostic value of coding gene expression signatures in JMML has previously been reported by Bresolin et al., ${ }^{15}$ we hypothesized that IncRNA expression might influence clinical outcome. Indeed, univariable Cox regression analysis identified 45 individual lncRNAs highly associated with OS $(P<0.001) \quad$ (Online Supplementary Table S6) of which 7 lncRNAs (UCKL1AS1, lnc-GPR63-5, lnc-ZSWIM2-3, Inc-RORB-1, IncNPFFR2-1, Inc-EMB-3 and lnc-CCDC90A-2) remained significantly associated with OS after multivariable Cox regression analysis with age category (< or $>2$ years old), HbF status, karyotype and molecular status $(P<0.001)$. Interestingly, these IncRNAs did not associate with known prognostic characteristics and pre-ranked GSEA could not identify a common (anti-)correlated gene set. We devised an IncRNA score, derived as a linear combination of the expression of the significant IncRNAs (method described in Garzon et al ${ }^{12}$ ) and dichotomized patients based on IncRNA score (scores below the median were classified as favorable and scores above as unfavorable). Patients with unfavorable lncRNA score had a significantly lower OS and EFS after HSCT (log rank test $P<0.001)$ (Figure $3 \mathrm{~A}$ and $\mathrm{C}$ ). Next, we compared the expression levels of 29,654 mRNA probes (21,659 unique genes) between patients with favorable and unfavorable IncRNA score. We identified 549 coding genes with differential expression between the two groups (absolute $\mathrm{FC} \geq 2$ ) (Figure $3 \mathrm{~B}$ ). Together, these results suggested that lncRNAs might be of prognostic significance in JMML. However, studies in independent patient cohorts are necessary to validate the prognostic value of IncRNA expression in JMML.

In conclusion, we documented for the first time the IncRNA landscape in $44 \mathrm{JMML}$ patients and have associated IncRNA expression with clinical and molecular characteristics. We demonstrated that JMML patients exhibit a distinct IncRNA expression profile compared to healthy controls and different JMML subgroups have distinct lncRNA signatures contributing to disease heterogeneity. Follow-up studies are needed to confirm the prognostic value of IncRNAs and to perform an integrative analysis of IncRNA expression profiles with mutational patterns, DNA methylation profiles and clinical patients' characteristics. This study paves the way for further functional research on the role of IncRNAs in JMML biology, and their diagnostic and therapeutic application.

Mattias Hofmans, ${ }^{1,2}$ Tim Lammens, ${ }^{1,3}$ Hetty H. Helsmoortel, ${ }^{4}$ Silvia Bresolin, ${ }^{5}$ Hélène Cavé, ${ }^{6}$ Christian Flotho, ${ }^{7}$

Henrik Hasle, Marry M. van den Heuvel-Eibrink, ${ }^{9,10}$

Charlotte Niemeyer, 7 Jan Stary, 11 Nadine Van Roy,

Pieter Van Vlierberghe, ,3an Philippé, $e^{, 3}$ and

Barbara De Moerloose $e^{1,3}$

${ }^{1}$ Department of Pediatric Hematology-Oncology and Stem Cell Transplantation, Ghent University Hospital, Belgium;

${ }^{2}$ Department of Clinical Chemistry, Microbiology and Immunology, Ghent University, Belgium; ${ }^{3}$ Cancer Research Institute Ghent, Belgium; ${ }^{4}$ Center for Medical Genetics, Ghent University Hospital, Belgium;
'Department of Women and Child Health, University of Padova, Italy; ${ }^{6}$ Department of Genetics, University Hospital of Robert Debré and Paris-Diderot University, Paris, France; 'Division of Pediatric Hematology and Oncology, Department of Pediatrics and Adolescent Medicine, University of Freiburg, Germany; ${ }^{8}$ Department of Pediatrics, Aarhus University Hospital Skejby, Aarhus, Denmark; ${ }^{9}$ Princess Máxima Center for Pediatric Oncology, Utrecht, the Netherlands; ${ }^{10}$ Dutch Childhood Oncology Group, The Hague, the Netherlands and "Department of Pediatric Hematology/ Oncology, Charles University and University Hospital Motol, Prague, Czech Republic

Funding: the authors would like to thank the Foundation against Cancer (2016-113, BDM), the Cancer Plan, action 29 (KP_29_020, $J P)$ and vzw Kinderkankerfonds - a non-profit childhood cancer foundation under Belgian law (grant to TL) for supporting this work. MH is a PhD candidate at Ghent University and this work is submitted in partial fulfilment of the requirement for the $P h D$.

Correspondence:mattias.hofmans@ugent.be doi:10.3324/haematol.2018.189977

Information on authorship, contributions, and financial \& other disclosures was provided by the authors and is available with the online version of this article at www. haematologica.org.

\section{References}

1. Niemeyer CM. RAS diseases in children. Haematologica. 2014; 99(11):1653-1662

2. Locatelli F, Niemeyer CM. How I treat juvenile myelomonocytic leukemia. Blood. 2015;125(7):1083-1090.

3. Stieglitz E, Taylor-Weiner AN, Chang TY, et al. The genomic landscape of juvenile myelomonocytic leukemia. Nat Genet. 2015; 47(11):1326-1333.

4. Caye A, Strullu M, Guidez F, et al. Juvenile myelomonocytic leukemia displays mutations in components of the RAS pathway and the PRC2 network. Nat Genet. 2015;47(11):1334-1340.

5. Helsmoortel HH, Bresolin S, Lammens T, et al. LIN28B overexpression defines a novel fetal-like subgroup of juvenile myelomonocytic leukemia. Blood. 2016;127(9):1163-1172.

6. Lipka DB, Witte $\mathrm{T}$, Toth $\mathrm{R}$, et al. RAS-pathway mutation patterns define epigenetic subclasses in juvenile myelomonocytic leukemia. Nature Commun. 2017;8(1):2126.

7. Stieglitz E, Mazor T, Olshen AB, et al. Genome-wide DNA methylation is predictive of outcome in juvenile myelomonocytic leukemia. Nature Commun. 2017;8(1):2127.

8. Volders PJ, Helsens K, Wang X, et al. LNCipedia: a database for annotated human IncRNA transcript sequences and structures. Nucleic Acids Res. 2013;41(Database issue):D246-251

9. Bhan A, Soleimani M, Mandal SS. Long Noncoding RNA and Cancer: A New Paradigm. Cancer Res. 2017;77(15):3965-3981.

10. Schwarzer A, Emmrich S, Schmidt F, et al. The non-coding RNA landscape of human hematopoiesis and leukemia. Nature Commun. 2017;8(1):218

11. Subramanian A, Tamayo P, Mootha VK, et al. Gene set enrichment analysis: a knowledge-based approach for interpreting genome-wide expression profiles. Proc Natl Acad Sci USA. 2005;102(43):1554515550.

12. Garzon R, Volinia S, Papaioannou D, et al. Expression and prognostic impact of IncRNAs in acute myeloid leukemia. Proc Natl Acad Sci USA. 2014;111(52):18679-18684.

13. Addelman S. The Generalized Randomized Block Design. Am Stat. 1969;23(4):35-36.

14. Lee S, Seo HH, Lee CY, et al. Human Long Noncoding RNA Regulation of Stem Cell Potency and Differentiation. Stem Cells Int. 2017;2017:6374504.

15. Bresolin S, Zecca M, Flotho C, et al. Gene expression-based classification as an independent predictor of clinical outcome in juvenile myelomonocytic leukemia. J Clin Oncol. 2010;28(11):1919-1927. 